\title{
Prediction of Difficult Airway Among Patients Requiring Endotracheal Intubation in a Tertiary Care Hospital in Eastern Nepal
}

\author{
Sindhu Khatiwada, ${ }^{1}$ Balkrishna Bhattarai, ${ }^{1}$ Krishna Pokharel, ${ }^{1}$ Roshan Acharya ${ }^{2}$ \\ 'Department of Anesthesiology and Critical Care, BPKIHS, Dharan, Nepal, ${ }^{2}$ Department of Otorhinolaryngology and Head \\ and Neck Surgery, Nobel Medical College, Biratnagar, Nepal.
}

\section{ABSTRACT}

Introduction: Various screening tests are done for predicting difficult laryngoscopy with variable diagnostic accuracy. Difficult laryngoscopy is being considered a surrogate indicator of difficult intubation, though it is not the exact measure of intubation difficulty. Our objectives were to find out the better predictor of difficult laryngoscopy amongst the routinely used tests and also to find the ability of difficult laryngoscopy to predict difficult intubation.

Methods: This prospective, observational study involved 314, ASA I/II adult patients requiring endotracheal intubation. Measurement of sternomental, thyromental and inter-incisor distances and gradings of mandibular protrusion and modified Mallampati were done. Statistical values including sensitivity and specificity of these tests were calculated to find the better predictor of difficult laryngoscopy. Cormack and Lehane laryngoscopy grade III/IV was defined as difficult laryngoscopy. Requirement of $>3$ attempts for endotracheal intubation was defined as difficult intubation.

Results: The sensitivity of the Modified Mallampatti Test for predicting difficult laryngoscopy was highest, $83 \%$ compared to other tests. Total 12 (3.8\%) patients had difficult laryngoscopy. Intubation was difficult in $7(2.2 \%)$ patients, of which four had difficult laryngoscopy $(\mathrm{P}<0.001)$.

Conclusions: Modified Mallampati test was better for predicting difficult laryngoscopy compared to other bedside screening tests. Difficult laryngoscop could significantly predict difficult intubation in our patients.

Keywords: airway evaluation; difficult intubation; difficult laryngoscopy; modified Mallampati test; sensitivity.

\section{INTRODUCTION}

Difficult intubation is an undesirable situation particularly if it is not anticipated. Modified Mallampati test, sternomental distance, thyromental distance, inter incisor gap and grades of mandibular protusion are some of the tests frequently performed for predicting difficult laryngoscopy and difficult intubation. ${ }^{1-3}$ The diagnostic accuracy of these screening tests is variably reported. ${ }^{4}$
Difficult laryngoscopy as graded by Cormack and Lehane has been considered a surrogate indicator of difficult intubation. ${ }^{5}$ Although a determining factor, poor glottis visualization is not the exact measure of intubation

Correspondence: Dr. Sindhu Khatiwada, Department of Anesthesiology and Critical Care, BPKIHS, Dharan, Nepal. Email: sindhukhatiwada@gmail.com, Phone: +977-9842045343. 
Khatiwad et al. Prediction of Difficult Airway among Patients Requiring Endotracheal Intubation in a Tertiary Care Hospital in Eastern Nepal

difficulty and its relation with difficult intubation may be variable in different patient population. ${ }^{6}$ The association between difficult laryngoscopy and difficult intubation in Nepalese patient has not been evaluated.

We aimed to find out the bedside screening test which is better at predicting difficult laryngoscopy amongst the routinely used tests and also to find the ability of difficult laryngoscopy to predict difficult intubation.

\section{METHODS}

This prospective observational study was conducted at the Department of Anesthesiology, BPKIHS, Dharan, Nepal from September 2012 to February 2013. Ethical approval from the institutional review committee and informed consent from patients was taken. Three hundred and fourteen consecutive patients, aged 1865 year, with ASA Physical Status I and II, requiring endotracheal intubation for various elective surgeries were included. Patients with diabetes, pregnancy or any obvious pathology involving the face, the neck and the airway were excluded.

Airway evaluation was done by the principal investigator in the preoperative patient holding area on the day of surgery. Sternomental distance (SMD) and thyromental distance (TMD) were measured with the head in extension and the mouth closed. Interincisor distance (IID) was measured with mouth fully open. Grades of mandibular protusion (MP) were noted as: Grade 1- lower incisor protruded anterior to upper incisor, Grade 2- Lower incisor brought edge to edge with upper incisor, Grade 3- Lower incisor could not be brought edge to edge. The airway class was assessed by using modified Mallampati test (MMT) by putting the patient in a sitting position, head in neutral, mouth fully open and the tongue maximally protruded out without phonation and the examiner eye to eye contact: ${ }^{7,8}$ Class $0=$ ability to see any part of the epiglottis upon mouth opening and tongue protrusion, Class $\mathrm{I}=$ soft palate, anterior and posterior pillars and uvula visualized, Class II = soft palate and uvula visualized, Class III = soft palate and base of uvula visualized, Class IV = only hard palate visualized. A value of SMD $<12 \mathrm{~cm}$, TMD $<6.5 \mathrm{~cm}$, IID $<3.5 \mathrm{~cm}, \mathrm{MP}$ grade 3 and modified Mallampati class (MMC) III or IV were considered as the predictors of difficult laryngoscopy. ${ }^{1}$

After induction of anaesthesia, direct laryngoscopy was performed using Macintosh number three blade with the patient's head in sniffing position. The attending anaesthesiologist performing laryngoscopy was unaware of the patient's airway measurements and grades. The laryngoscopy view was graded according to the Cormack and Lahane $(C L),{ }^{5}$ as follows: Grade
I-visualization of entire glottic aperture, Grade IIvisualization of only posterior aspects of the glottic aperture, Grade III- visualization of only tip of epiglottis, Grade IV- visualization of only the soft palate. Grade III or IV of laryngoscopic view was defined as difficult laryngoscopy and assumed as the predictor of difficult intubation.

During intubation, an anaesthesia technician noted the number of attempts and the alternate techniques used by the attending anaesthesiologist. Requirement of more than three attempts for proper insertion of the tracheal tube with conventional laryngoscopy was defined as difficult intubation. ${ }^{9}$ External laryngeal manipulation was allowed to facilitate intubation only after assessment of the laryngoscopic view. The alternate techniques included use of stylet or bougie, patient repositioning, change of operator or blade or tracheal tube or laryngoscope and were applied according to the discretion of the attending anaesthesiologist.

\begin{tabular}{|ll|}
\hline \multicolumn{2}{|l|}{ Table 1. Characteristics of patient. } \\
\hline Parameters & Values \\
Age $(\mathrm{yr})$ & $38.2 \pm 14.5$ \\
Sex ratio (M/F) & $213 / 101$ \\
Weight $(\mathrm{kg})$ & $54.3 \pm 10.2$ \\
Height $(\mathrm{m})$ & $1.57 \pm .07$ \\
Body mass index $\left(\mathrm{kg} / \mathrm{m}^{2}\right)$ & $21.8 \pm 2.4$ \\
Sternomental distance $(\mathrm{cm})$ & $14.9 \pm 2.4$ \\
Thyromental distance $(\mathrm{cm})$ & $7.9 \pm 5.7$ \\
Inter-incisor distance $(\mathrm{cm})$ & $4.9 \pm 0.7$ \\
Mandibular protrusion grade, & $312 / 2$ \\
$<3 / 3$ & \\
Modified Mallampati class, 0/I/ & $2 / 104 / 108 / 99 / 1$ \\
II/III/IV & \\
Laryngoscopic view, I/II/III/IV & $177 / 125 / 12 / 0$ \\
\hline
\end{tabular}

Values are presented as mean $\pm S D$ or number of patients.

The collected data was entered in Microsoft Excel 2007 and analyzed with Statistical Package for Social Science (version 11.5 PC + for Windows; SPSS). Numerical variables are presented as mean $\pm \mathrm{SD}$. Categorical variables are presented as the number (\%) of patients. Independent t-test was applied to compare continuous variables. Chi-square test was applied to compare categorical variables. Statistical values including sensitivity, specificity, positive predictive value (PPV), negative predictive value (NPV) and accuracy of the various tests for difficult laryngoscopy was calculated. A $P$ value of $<0.05$ was considered as statistically significant.

\section{RESULTS}

Modified Mallampati class III and IV was found in $100(31.8 \%)$ patients. Total $12(3.8 \%)$ patients had 
Khatiwad et al. Prediction of Difficult Airway among Patients Requiring Endotracheal Intubation in a Tertiary Care Hospital in Eastern Nepal

grade III laryngoscopic view and none had a grade of IV (Table 1). Out of 12 patients with difficult laryngoscopy 10 had modified Mallampati grade III or IV $(\mathrm{P}<0.001)$ (Figure 1). The sensitivity of MMT for predicting difficult laryngoscopy was $83 \%$ and the negative predictive value was $99 \%$ (Table 2). Total $7(2.2 \%)$ patients required more than three attempts for intubation. Among the patients who were difficult to intubate, stylet was used in two patients, Mc-Coy laryngoscope was used in two patients and both stylet use and change of operator was done in two patients. Intubation was successful in all the patients. Four out of seven patients with difficult intubation had grade III laryngoscopic view $(P<0.001)$ (Figure 2). Patients who were difficult to intubate had higher, III/IV Mallampati grades $(\mathrm{P}<0.001)$. The mean age $(48.2 \pm 11.7 \mathrm{VS}$. $37.8 \pm 14.5, P=0.014)$ and the mean Mallampati score $(2.8 \pm 0.3$ vs. $1.9 \pm 0.8, P<0.001)$ of patients with difficult laryngoscopy was high compared to patients with easy laryngoscopy.

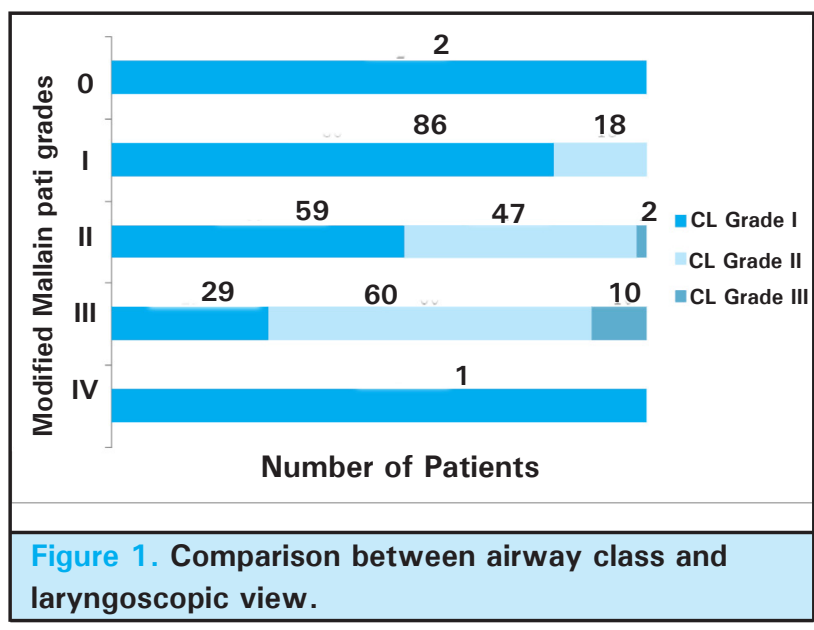

\begin{tabular}{|c|c|c|c|c|c|}
\hline Parameters & SMD $<12 \mathrm{~cm}$ & TMD $<6.5 \mathrm{~cm}$ & IID $<3.5 \mathrm{~cm}$ & $\begin{array}{l}\text { MP grade } \\
\text { III }\end{array}$ & $\begin{array}{l}\text { MMC } \\
\text { III/IV }\end{array}$ \\
\hline Frequency (\%) & $25(7.9)$ & $90(28.6)$ & $2(0.6)$ & $2(0.6)$ & $100(31.8)$ \\
\hline True positive & 1 & 2 & 1 & 1 & 10 \\
\hline False positive & 24 & 88 & 1 & 1 & 90 \\
\hline True negative & 276 & 214 & 301 & 301 & 212 \\
\hline False negative & 11 & 10 & 11 & 11 & 2 \\
\hline Sensitivity (\%) & 8.3 & 16.6 & 8.3 & 8.3 & 83.3 \\
\hline Specificity (\%) & 92 & 70 & 99 & 99 & 70 \\
\hline Positive predictive value (\%) & 3.4 & 2.2 & 50 & 50 & 10 \\
\hline Negative predictive value (\%) & 96 & 95.5 & 96 & 96 & 99 \\
\hline Accuracy (\%) & 88 & 68.7 & 96 & 96 & 70.7 \\
\hline
\end{tabular}

Values are presented as number of patients or percentage. SMD = sternomental distance; TMD $=$ thyromental distance; IID $=$ interincisor distance; $M P=$ mandibular protrusion; $M M C=$ modified Mallampati class

\section{DISCUSSION}

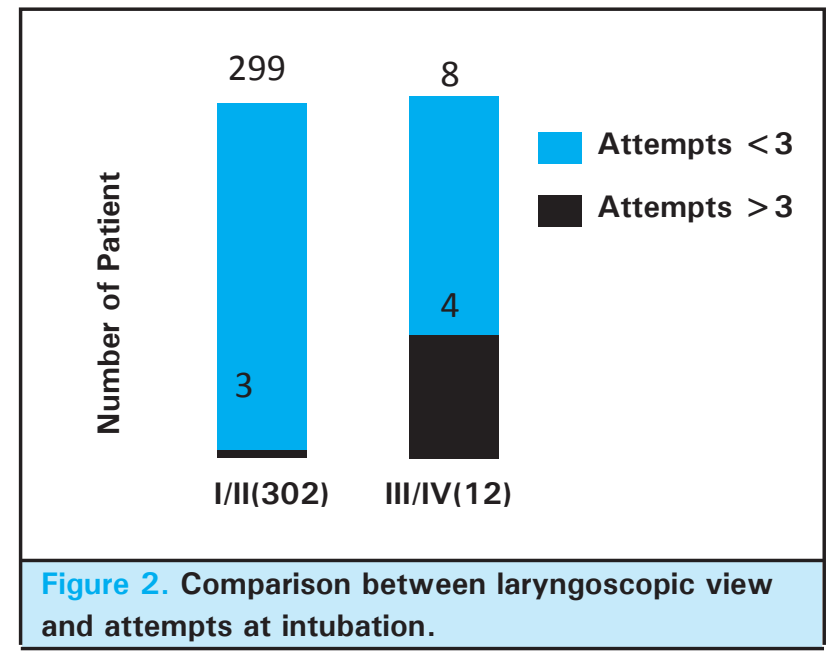

Modified Mallampati test, because of its highest sensitivity is better compared to other routinely used tests for predicting difficult laryngoscopy. Difficult laryngoscopy significantly predicts difficult intubation in our patients.

An airway physical examination using various tests is recommended whenever feasible, before the initiation of anaesthetic care, and is routinely being practiced in all patients. ${ }^{10}$ The variable diagnostic accuracy of these screening tests for predicting difficult airway probably results from the differences in the incidence of difficult intubation, inadequate statistical power, different test thresholds, or differences in patient characteristics. ${ }^{3,4}$

When the statistical values of the routinely used tests for predicting difficult laryngoscopy was calculated, 
MMT had the highest sensitivity in our patients. Higher sensitivity and good accuracy of MMT for predicting both difficult laryngoscopy and difficult intubation has been reported. ${ }^{4,11}$ Patients with higher MMC had difficult laryngoscopy and required increased attempts for intubation in our study and this finding is in accordance with the earlier reports. ${ }^{8,12,13}$ However, MMC did not have significant correlations with $\mathrm{CL}$ grade and the number of attempts at endotracheal intubation. ${ }^{14,15}$ Similarly, the pooled sensitivity of MMT for predicting difficult laryngoscopy and difficult intubation was only $49 \%$ and $35 \% .4,16$

The TMD, SMD, MP, and IID failed to reach high sensitivity as a predictor of difficult laryngoscopy in our study. The limited clinical value and lower sensitivity of these bedside screening tests for predicting difficult laryngoscopy or intubation has been reported..$^{3,4}$ In contrast, TMD, IID and SMD were the significant predictors of difficult laryngoscopy or difficult intubation. ${ }^{17-19}$

Although, the sensitivity, specificity and NPV of MMT for predicting difficult laryngoscopy were high in our patients, its positive predictive value was very low. However, because of its very high sensitivity, we still regard modified Mallampati as a valuable bedside screening test for airway assessment with direct laryngoscopy in our patient population. We concur with Arne et al. that decreasing the prediction of false negative cases is far more important than diagnosing false positive cases. ${ }^{12}$

When the characteristics of our patients were compared, the patients with difficult laryngoscopy were significantly older and had higher Mallampati score compared to patients with easy laryngoscopy. A significant association between older patients and higher Mallampati grade with difficult laryngoscopy and intubation has been reported. ${ }^{12,20}$

The prevalence of difficult laryngoscopy was 3.8\% in our patients. Similar prevalence of $4.9 \%$ was found in an earlier study done in Nepalese population. ${ }^{21}$ Whereas, a higher prevalence of $9.7 \%$ was found in Indian population. ${ }^{20}$ Wide variations in the prevalence of difficult laryngoscopy ranging from $2 \%$ to $27 \%$ has been reported, and could be due to the differences in the characteristics of the studied patients. ${ }^{11}$

Variations in the definition of difficult intubation exist and could be the reason for differences in the prevalence of difficult intubation. Poor glottis visualization is considered as the surrogate indicator of difficult intubation. ${ }^{4,5,16}$ However, adequate glottis visualization during laryngoscopy is not sufficient to guarantee the advancement of the tube between the vocal cords and into the trachea. ${ }^{3}$ The intubation difficulty scale (IDS) comprising of seven airway related parameters have been proposed for determining the difficulty during intubation. ${ }^{22}$ Similarly, Arne et al. defined difficult intubation as requiring unusual laryngoscope or techniques including gum elastic bougies and fibreoptic intubation. ${ }^{12}$

To make it simple we choose the definition given by the American Society of Anesthesiologists task force, the multiple attempts at intubation as criteria in our study. ${ }^{9}$ The prevalence of difficult intubation was $2.2 \%$ in our patients and is similar to the prevalence of $1.4 \%^{1,23}$ and $2.4 \%,{ }^{24}$ as reported earlier. A higher prevalence ranging from $4 \%$ to $6 \%$ is reported with the use of IDS. ${ }^{20,22}$ We found direct association between poor laryngoscopic view and increase in the number of attempts at intubation in our study and this finding is similar to the earlier reports. ${ }^{25}$

Overall, MMT is a better predictor of difficult laryngoscopy as compared to other routinely used tests. However, when used alone it may not be adequate for confidently predicting difficult laryngoscopy or tracheal intubation and therefore should form a part of the overall assessment of the airway. 4,12,17 The difficult laryngoscopic views significantly predicts difficult intubation.

A small sample size is one of our limitations. Also, as laryngoscopy and intubation was performed by the attending anaesthesiologist, we do assume some interindividual variability in the laryngoscopic grading and in the attempts of intubation.

\section{CONCLUSIONS}

Modified Mallampati test, because of its highest sensitivity is the better predictor of difficult laryngoscopy compared to other routinely used tests. Difficult intubation can be predicted by difficult laryngoscopy. Assessment of modified mallampati test and laryngoscpic grades is valuable for predicting difficult laryngoscopy and intubation in our patient population.

\section{Conflict of Interest: None.}




\section{REFERENCES}

1. Cattano D, Panicucci E, Paolicchi A, Forfori F, Giunta F, Hagberg C. Risk factors assessment of the difficult airway: An Italian survey of 1956 patients. Anesth Analg. 2004 Dec;99(6):1774-9. [uuMed | DOI]

2. Tuzuner-Oncul AM, Kucukyavuz Z. Prevalence and prediction of difficult intubation in maxillofacial surgery patients. J Oral Maxillofac Surg. 2008 Aug;66(8):1652-8. [PubMed | DOI]

3. Vannucci A, Cavallone LF. Bedside predictors of difficult intubation: a systematic review. Minerva Anestesiol. 2016 Jan;82(1)69-83. [pubMed]

4. Shiga T, Wajima Z, Inoue T, Sakamoto A. Predicting difficult intubation in apparently normal patients: a meta-analysis of bedside screening test performance. Anesthesiology. 2005 Aug;103(2):429-37. [PubMed]

5. Cormack RS, Lehane J. Difficult tracheal intubation in obstetrics. Anaesthesia. 1984 Nov; 39(11):1105-11. [․ PubMed]

6. Juvin P, Lavaut E, Dupont H, Lefevre P, Demetriou M, Dumoulin JL, Desmonts JM. Difficult tracheal intubation is more common in obese than in lean patients. Anesth Analg. 2003 Aug;97(2):595-600. [PubMed]

7. Samsoon GL, Young JR. Difficult tracheal intubation: a retrospective study. Anaesthesia. 1987 May;42(5):487-90. [pubMed]

8. Ezri T, Warters RD, Szmuk P, Saad-Eddin H, Geva D, Katz J et al. The incidence of class "zero" airway and the impact of Mallampati score, age, sex, and body mass index on prediction of laryngoscopy grade. Anesth Analg. 2001 Oct;93(4):1073-5. [․ㅏbMed]

9. ractice guidelines for management of the difficult airway. A report by the American Society of Anesthesiologists Task Force on Management of Difficult Airway. Anesthesiology. 1993 Mar;78(3):597-602. [FullText]

10. Practice guidelines for management of the difficult airway. An updated report by the American Society of Anesthesiologists Task Force on Management of Difficult Airway. Anesthesiology. 2013 Feb;118(2):251-70. [FullText]

11. Lee A, Fan LT, Gin T, Karmakar MK, Ngan kee WD. A systematic review (meta-analysis) of the accuracy of the Mallampati tests to predict the difficult airway. AnesthAnalg. 2006 Jun;102(6):1867-78. [PubMed | DOI]

12. Arne J, Descoins P, Fusciardi J, Ingrand P, Ferrier B, Boudigues D et al. Preoperative assessment for difficult intubation in general and ENT surgery: predictive value of clinical multivariate risk index. Br J Anaesth. 1998 Feb;80(2):140-6. [PubMed]

13. Mallampati SR, Gatt SP, Gugino LD, Desai SP, Waraksa B, Freiberger $\mathrm{D}$ et al. A clinical sign to predict difficult tracheal intubation: a prospective study. Can Anaesth Soc J. 1985 Jul;32(4):429-34. [pubMed]
14. Wanderley GHS, Lima LC, de Menezes Couceiro TC, Silva WV, Coelho RQGA, Lucena ACC et al. Clinical criteria for airway assessment: Correlations with laryngoscopy and endotracheal intubation conditions. Open Journal of Anesthesiolog. 2013;3:320-5. [FullText]

15. Hester CE, Dietrich SA, White SW, Secrest JA, Lindgren KR, Smith T. A comparison of preoperative airway assessment techniques: the modified Mallampati and the upper lip bite test. AANA J. 2007 Jun;75(3):177-82. [․ㅏbMed]

16. Lundstøm LH, Vester-Andersen M, Møller AM, Charuluxananan S, L'hermite J, Wetterslev J; Danish Anaesthesia Database. Poor prognostic value of the modified Mallampati score: a meta-analysis involving 177088 patients. Br J Anaesth. 2011 Nov;107(5):659-67. [PubMed | DOI ]

17. Fritscherova S, Adamus M, Dostalova K, Koutna J, Hrabalek L, Zapletalova J et al. Can difficult intubation be easily and rapidly predicted? Biomed Pap Med Fac Univ Palacky Olomouc Czech Repub. 2011 Jun;155(2):165-71. [PubMed]

18. Aktas S, Atalay YO, Tugrul M. Predictive value of bedside tests for difficult intubations. Eur Rev Med Pharmacol Sci. 2015;19(9):1595-9. [PubMed]

19. Savva D. Prediction of difficult tracheal intubation. Br J Anaesth. 1994 Aug;73(2):149-53. [PubMed]

20. Prakash S, Kumar A, Bhandari S, Mullick P, Singh R, Gogia AR. Difficult laryngoscopy and intubation in the Indian population: An assessment of anatomical and clinical risk factors. Indian J Anaesth. 2013 Nov;57(6):569-75. [FullText |DOI]

21. Shah S. Prevalence and prediction of difficult intubation in Nepalese population. JSAN. 2015;2:17-20. [FullText | DOI]

22. Adnet F, Borron SW, Racine SX, Clemessy JL, Fournier JL, Plaisance $\mathrm{P}$, et al. The intubation difficulty scale (IDS): proposal and evaluation of a new score characterizing the complexity of endotracheal intubation. Anesthesiology. 1997 Dec;87(6):1290-7. [PubMed]

23. Khan ZH, Eskandari S, Yekaninejad MS. A comparison of the Mallampati test in supine and upright positions with and without phonation in predicting difficult laryngoscopy and intubation: A prospective study. J Anaesthesiol Clin Pharmacol. 2015 Apr-Jun;31(2):207-11. [

24. L Hermite J, Nouvellon E, Cuvillon P, Fabbro-Peray P, Langeron $\mathrm{O}$, Ripart J. The simplified predictive intubation difficulty score: a new weighted score for difficult airway assessment. Eur J Anaesthesiol. 2009 Dec;26(12):1003-9. [pubMed]

25. Rabadi D, Baker AA, Al-Qudah M. Correlation between oro and hypopharynx shape and position with endotracheal difficulty. Rev Bras Anestesiol. 2014 Nov-Dec;64(6):433-7. [PubMed | DOI] 